\title{
NUMERICAL AND EXPERIMENTAL STUDY ON MECHANICAL BEHAVIOUR OF THE AISi10Mg ALUMINIUM STRUCTURES MANUFACTURED ADDITIVELY AND SUBJECTED TO A BLAST WAVE
}

\author{
M. Stanczak ${ }^{1 *}, 3$, T. Fras $^{1}$, L. Blanc ${ }^{1}$, P. Pawlowski ${ }^{2}$, and A. Rusinek ${ }^{3}$
}

\footnotetext{
${ }^{1}$ Department of Protection Technologies, Security \& Situational Awareness, French-German Research Institute of Saint-Louis (ISL), 68301 Saint-Louis, France

${ }^{2}$ Institute of Fundamental Technological Research Polish Academy of Sciences, Polish Academy of Sciences (PAS) 02-106 Warsaw, Poland

${ }^{3}$ Laboratory of Microstructure Studies and Mechanics of Materials, LEM3 Laboratory, Lorraine University, 57070 Metz, France
}

\begin{abstract}
The paper is related to energy absorptive properties of additively manufactured metallic cellular structures. The samples of Honeycomb, Auxetic, rhomboidal Lattice and a regular Foam are subjected to a dynamic compression due to the blast tests. The cuboidal samples are manufactured by the Direct Metal Laser Sintering (DMLS) method using AlSi10Mg aluminium powder. The experimental tests are performed by means of an Explosive Driven Shock Tube (EDST). The measured results of the transmitted forces in relation to the shortening of the samples allow to analyse the deformation processes of each selected geometry. In addition, the evaluation of the structural responses leads to identification of the structure properties, such as the equivalent stress over equivalent strain or the energy absorption per a unit of mass. Moreover, the process of compression is modelled numerically using the explicit code LS-DYNA R9.0.1. The obtained simulations provide the complete analysis of the experimentally observed mechanisms.
\end{abstract}

\section{Introduction}

Nowadays, numerous research studies focus on improvements of protection systems against impacts or explosions, e.g. [1-5]. Modern and advanced techniques of manufacturing, like an additive manufacturing (AM) may be used to produce structures with optimized geometrical properties. The AM methods provide a freedom in designing geometry or defining density of manufactured samples. Thanks to additive manufacturing, spare parts adaptable to already existing components may be fast and efficiently produced. However, the behaviour of structures produced due to the AM technique is complex and it still requires investigations. A particular attention should be focused on the behaviour of AM structures affected by an 
explosion. So far, only several works refer to dynamic responses of AM samples in blast conditions [6-7].

In consequence, the hereby discussed experimental tests, performed by means of the Explosive Drive Shock Tube (EDST) test stand, concern four different AM structures under an influence of an explosive wave. To compare the absorptive properties, two structures consisting of 2D unit cells (Honeycomb and Auxetic) and two others characterized by a 3D geometry (rhomboidal Lattice and regular Foam) are selected. The studies are based on the printed cubic samples without any additional post-treatment, often called 'as built' [8]. An analysis of the deformation and damaged processes is realized due to explicit simulations in LS-DYNA R9.0.1.

\section{Material characteristics and manufacturing process}

The tested aluminium AlSi10Mg samples are additively manufactured by the Direct Metal Laser Sintering (DMLS) method applied in the EOSINT M 280 3D printer. The DMLS is based on the selective sintering of an evenly distributed powder on the build platform. The $30 \mu \mathrm{m}$ powder layer is melted according to a bidirectional scanning strategy at an angle of $67^{\circ}$ by a $370 \mathrm{~W}$ laser beam. The printing technique contributes to the formation of a fine microstructure during the solidification process and unification the macro properties [9]. The settings during manufacturing, a type of the powder, as well as the sample position in relation to the build platform or the final treatment may significantly affect the properties of the obtained product [10].

In order to determine the material characteristics of AlSi10Mg aluminium, the quasi-static and dynamic compression tests has been carried out. The performed tests are based on the samples printed horizontally and vertically with respect to the build platform. Figure 1 shows the obtained results of the true stress over the plastic strain. The tests are performed at the strain rate between $0.001 / \mathrm{s}-3300 / \mathrm{s}$ and at the temperature range of $293 \mathrm{~K}-473 \mathrm{~K}$. An adiabatic-isothermal correction of the registered stress-strain material curves is introduced to the results at high strain rates, according to [11].

The results indicate that the print direction affect the material response. The vertically printed samples exhibit the highest values of the stress in the first deformation stage (up to 0.25 strain). However, the horizontally printed samples exhibit highest hardening in second regime. It is observed that the $\mathrm{AlSi} 10 \mathrm{Mg}$ aluminium is only slightly sensitive to the strain rate changes, however the temperature increase causes a stronger material softening.

Table 1. The tested structures and their geometrical properties.

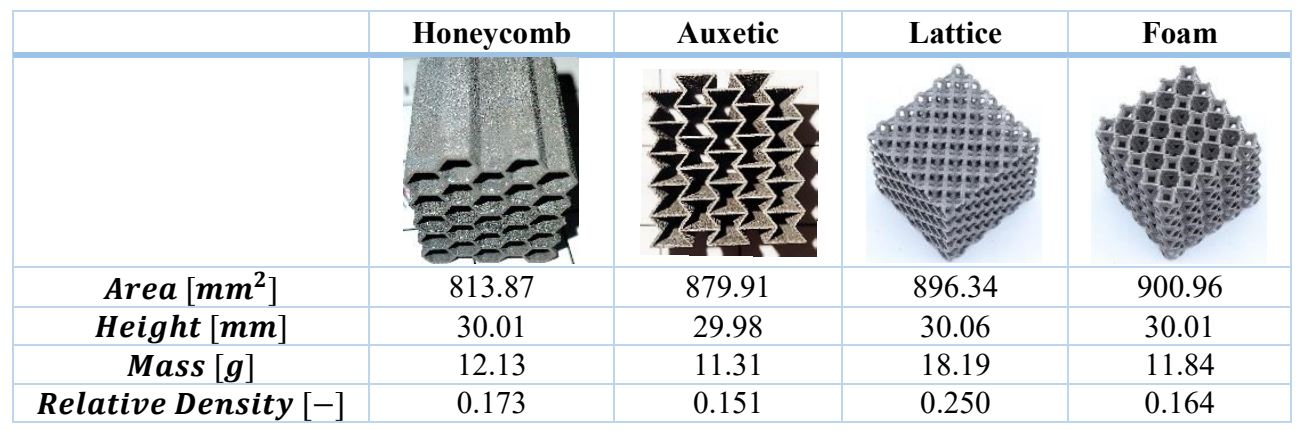

The objective of the study is a comparison of the absorptive properties of four different geometries: Honeycomb, Auxetic, Lattice and regular Foam (Table 1). The Honeycomb and Auxetic represent the structures with 2D unit cells and Lattice and Regular Foam are 
characterized by three-dimensional (3D) geometries. The tests were based on the 'as built' cubic samples with the edge length of $30 \mathrm{~mm}$. The samples are characterized by a similar relative density equalled $0.2 \pm 0.05$.

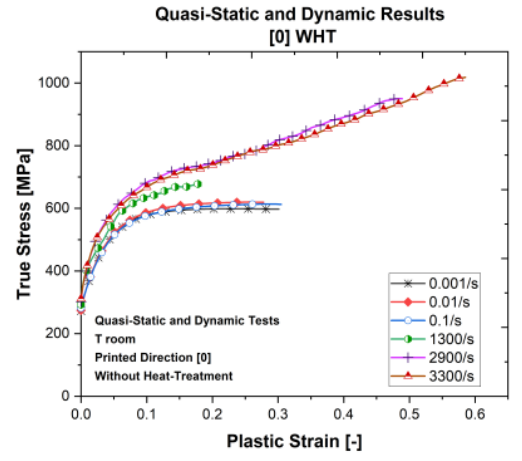

a)

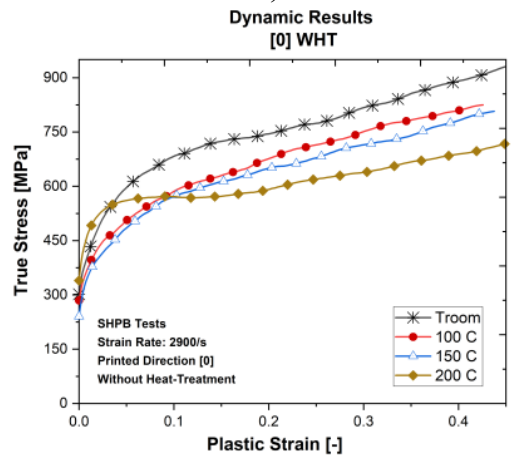

c)

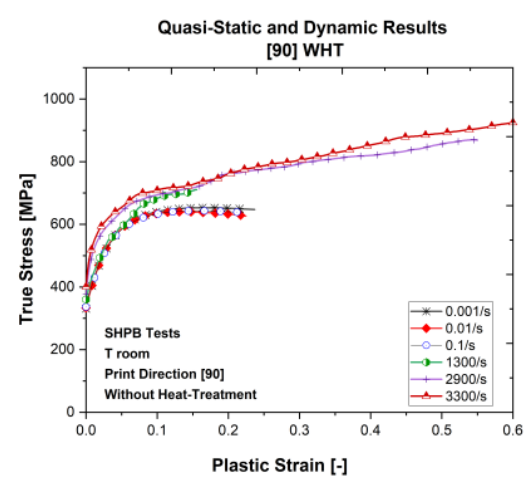

b)

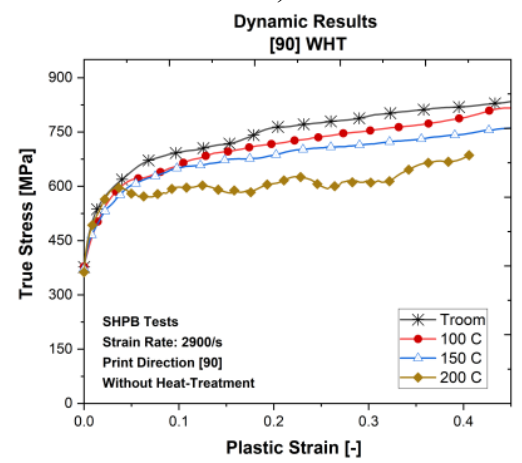

d)

Fig. 1. The experimental material test results describing the horizontally and vertically printed samples at different strain rates $(a, b)$ and at different temperatures $(c, d)$.

\section{Experimental set-up}

The manufactured samples are subjected to blast loadings using an Explosive Driven Shock Tube (EDST), presented in Figure 2a-b. This experimental set-up consists of the $1.75 \mathrm{~m}$ long shock tube, in front of which $30 \mathrm{~g}$ of the C-4 explosive charge is located. An explosion generates a spherical wave which travels through the tube. Due to internal reflections and the travelled distance, the blast wave forms a planar front at the end of the tube. The final blast loadings applied to the front plate depends on the mass of the charge and the length of the tube. Their measured profiles are presented in Figure 2c [12].

The movement of the front steel plate accelerated by the explosive wave causes a compression of the tested samples. During the damage process, the force transmitted to the back plate is measured by a PCE 206C piezoelectric force sensor, Figure $2 \mathrm{~b}$. In addition, a high-speed camera Phantom V311 records the whole deformation process. Then, the Digital Image Correlation (DIC) allows to obtain the displacement of the front plate over the time. The correlation of the force and the displacement enables to calculate the stress $\sigma_{e q}(\mathrm{t})$ over the strain $\varepsilon_{e q}(\mathrm{t})$ for each sample according to the Equations 1 and 2. 


$$
\begin{gathered}
\sigma_{e q}(\mathrm{t})=\frac{\mathrm{F}_{\text {trans }}(\mathrm{t})}{\mathrm{A}_{\text {ref }}} \\
\varepsilon_{e q}(\mathrm{t})=\frac{\mathrm{x}(\mathrm{t})}{\mathrm{L}_{0}}
\end{gathered}
$$

where: $F_{\text {trans }}(t)$ - total force transmitted to the back plate, $A_{\text {ref }}$ - reference cross-section area of the sample, $x(t)$ - displacement of the front plate, $L_{0}$ - initial height of the sample.

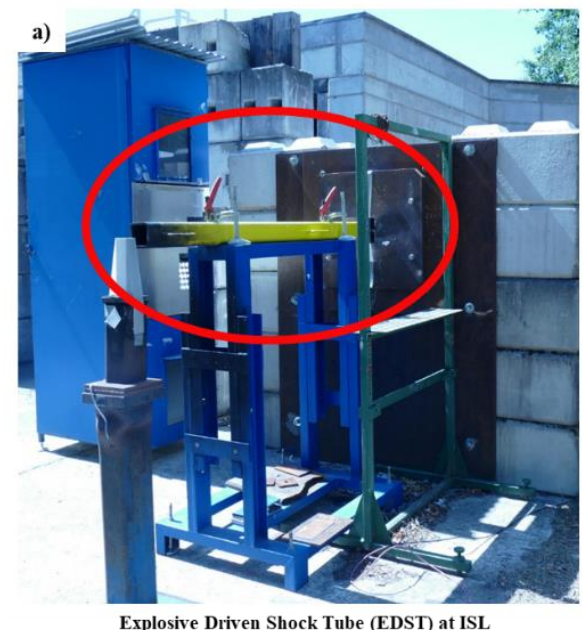

Explosive Driven Shock Tube (EDST) at ISL

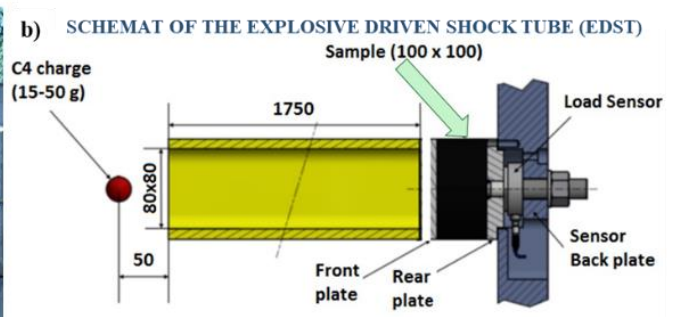

c)

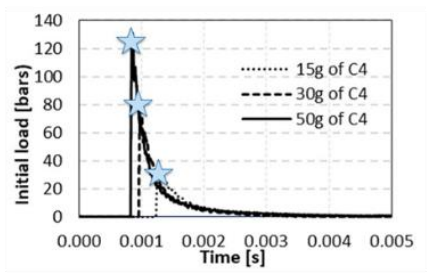

Fig. 2. The test stand of the Explosive Driven Shock Tube: a) side view, b) schema of the EDST, c) initial impulse profiles of the load dependent on the used charges.

\section{Results}

Typically, the response of a compressed metallic cellular structures may be described by three main phases [13-14], which is also observed for the tested structures. The elastic response of cellular structures ends with a first stress peak. This is often the maximum obtained value before the total damage of the sample. Then the deformation follows a plateau regime, in which the registered force oscillates around a certain value. In this region, the structure undergoes the plastic deformation such as bending or stretching. Finally, a sudden sharp rise in the obtained stress corresponds to a fully damaged structure.

Figure 3 presents the summary of the obtained results of the stress over the strain for Honeycomb, Auxetic, Lattice and Foam. It can be seen that each sample is characterized by the peak stress and plateau regime. However, Lattice does not exhibit the densification phase. It means that the sample absorbs the whole generated load before its full damage. The Foam structure is also characterized by a high value of the transmitted force, which results in the plateau stress of 7.8 $\mathrm{MPa}$. The structures composed with the $2 \mathrm{D}$ unit cells turn out to be the least efficient, wherein the plateau stress of Auxetic (equal to $1.44 \mathrm{MPa}$ ) is more than two times lower than that of the Honeycomb structure (3.37 MPa). 


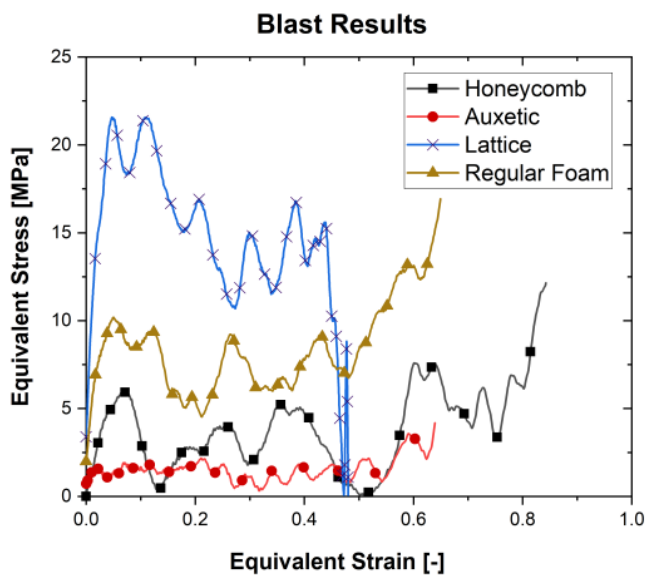

Fig. 3. The experimental blast results for the tested structures of Honeycomb, Auxetic, Lattice and Foam, performed by means of the EDST with $30 \mathrm{~g}$ of the C4 explosive charge.

The results are correlated with the damage process registered by a high-speed camera (Figure 4). It can be seen that Honeycomb and Auxetic exhibit a progressive, brittle-type damage as the cells collapse row-by-row. Wherein, the Honeycomb's cells deform in an outward direction, enlarging the observed surface transversely to the applied load. The cells of Auxetic collapse to the middle of the structure. The observed process proved the typical behaviour of the structures with a negative Poisson ratio. The Lattice and Foam exhibit strong elastic - plastic deformations. Both structures expand in the direction perpendicular to the applied load, along with the height reduction.

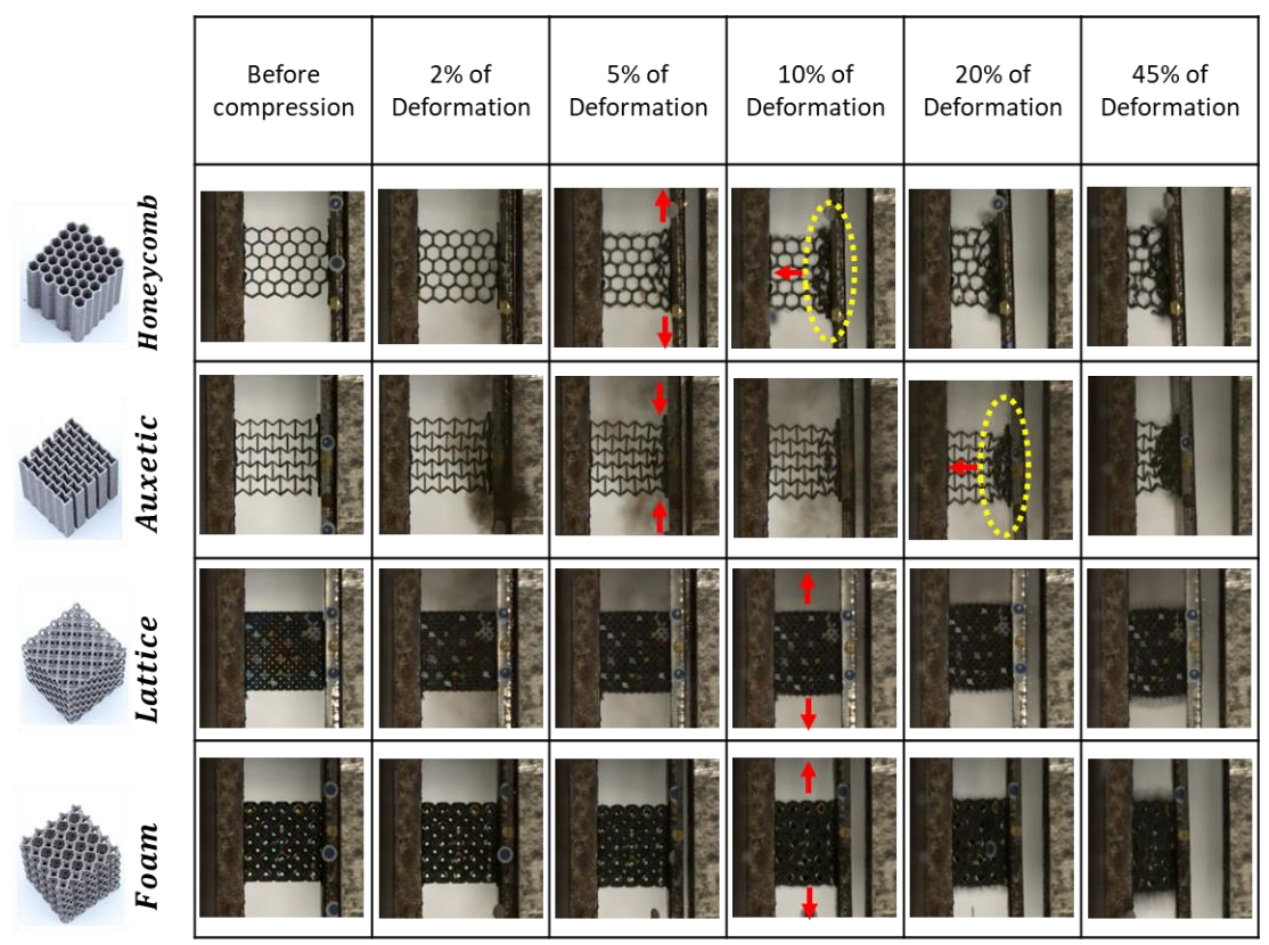

Fig. 4. The subsequent frames recorded by a high-speed camera during the performed blast tests. 


\section{Numerical approach}

In order to analyse the damage process in details, the explicit simulation in the LS-DYNA R9.0.1 is developed. The experimental set up is prepared in Ls-PrePost and consists of the Lagrange solid parts of the tested sample, placed between two steel plates. The contact between the components is obtained by the function *AUTOMATIC_SURFACE_TO_SURFACE. The crushing load is assigned to the nodes of the upper surface of the front plate.

The in-house obtained characteristics of the AlSi10Mg aluminium alloy are used to describe the material properties of the tested structure (see Section 2). To introduce the experimental curves, the *MAT_Linear_Pleasticity 024 is chosen to simulate the material behaviour of structures. The *MAT_Simplified_Johnson_Cook 098 is used to describe the material behaviour of the steel blocks. *Mat_098 is a simplified Johnson-Cook constitutive function which does not account for temperature effects and is decoupled from the JohnsonCook fracture model [16]. A maximum effective strain at failure of 0.1 is used to simulate a brittle damage. The bottom surface of the second block is fully constrained, simulating the experimental bunker wall. A more exact description of the numerical configuration is presented in $[12,16]$.

a)

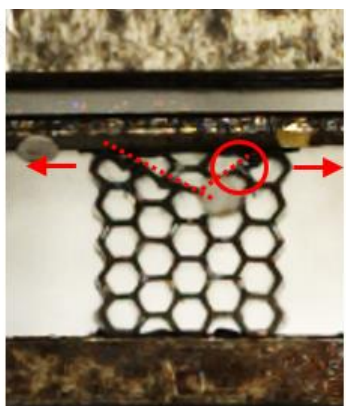

d)

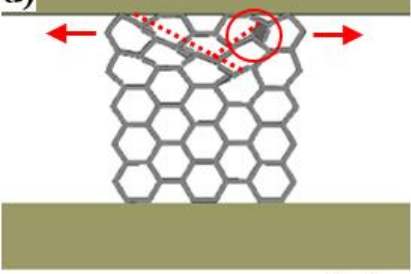

\section{g)}

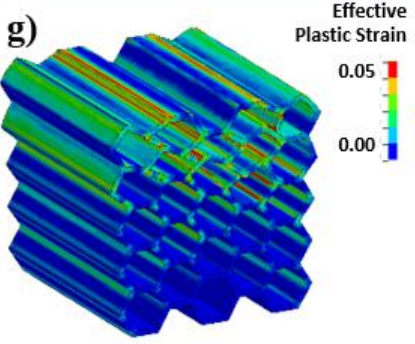

b)

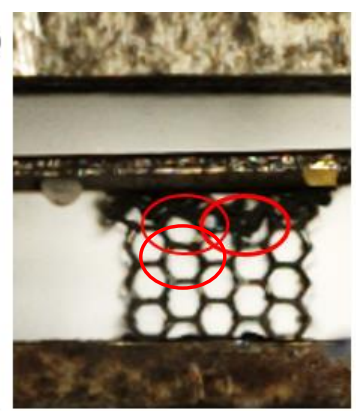

e)
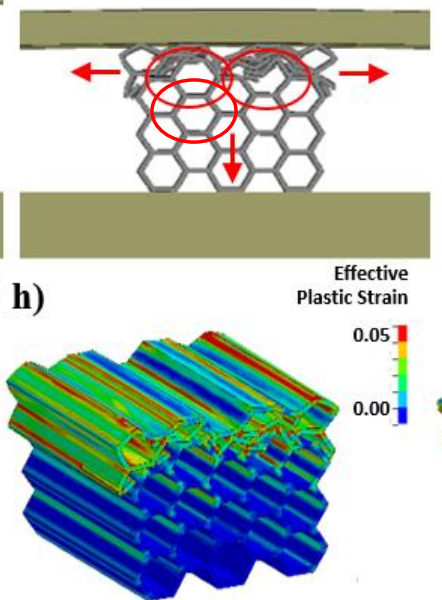

c)

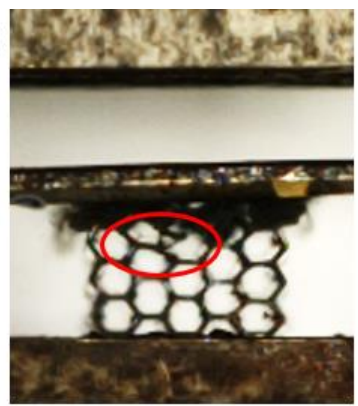

f)

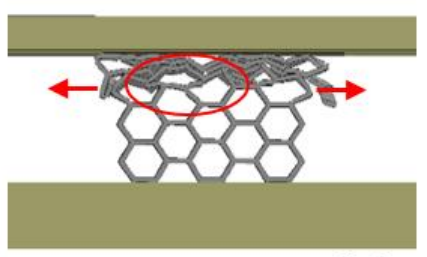

i)

Effective

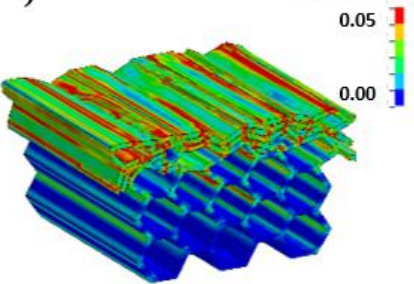

Fig. 5. The comparison of the blast experiment of the Honeycomb structure $(a, b, c)$ with its numerical simulation: front view $(\mathrm{d}, \mathrm{e}, \mathrm{f})$ and the isometric view presented in maps of the effective plastic strain $(\mathrm{g}, \mathrm{h}, \mathrm{i})$. 
Figure 5 presents the comparison between the blast test results for the Honeycomb structure alongside with the developed simulation. Figures $5 \mathrm{~d}-\mathrm{f}$ show a progressive damage of cells (row-by-row) that is also observed during the experimental test. The cells bend and stretch outwards the structure. The simulation allows to indicate the most strained and damaged walls of the structure, which may be difficult to observe during the test. The deformation explains the differences in the strained cells mod at the same row level (the red circles at Figures 5b-e).

In addition, Figures $5 \mathrm{~g}$-i indicate the areas which undergo the strongest strain during the compression. In the undeformed zones, the geometry may be optimized without affecting the response of the structure.

\section{Discussion}

The results from the performed quasi-static and dynamic tests are referred to the mass of each sample. The specific energy absorption (SEA) is determined according to the Equation 3.

$$
S E A=\frac{E A}{m}=\frac{\int_{0}^{x} d F(x) d x}{m}
$$

where: EA - absorbed energy, $\mathrm{m}$ - mass of the sample, $\mathrm{F}(\mathrm{x})$ - registered force and $\mathrm{x}$ - change in height of the sample.

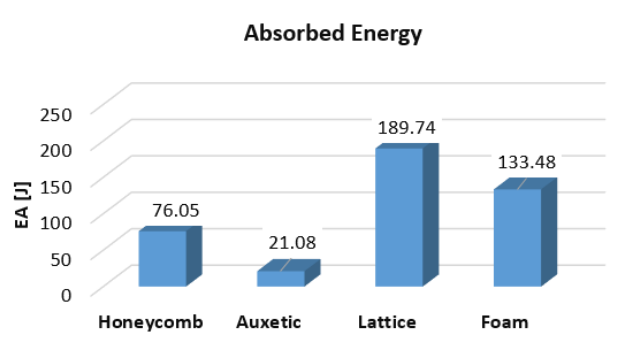

a)

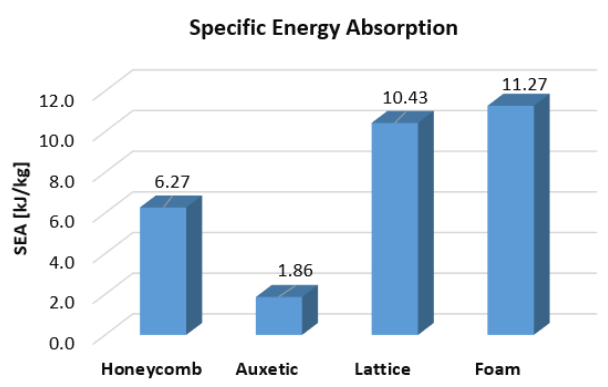

b)

Fig. 6. a) Absorbed energy and b) specific energy absorption of each sample.

The resulted absorbed energy (Figure 6a) is in line with the trend shown in the stressstrain diagram (Figure 3). The Lattice structure absorbs most energy during the performed explosive test. The Foam, in contrast to the Auxetic structure $(E A \approx 21 J)$, is proven also to have a high absorptive properties $(E A \approx 133 J)$. However, when the mass of structures is taken into account, both samples with 3D unit cells exhibit similar values of the SEA (close to $11 \mathrm{~kJ} / \mathrm{kg}$ ), which are higher than the characteristics of the 2D-cells structures Figure $6 \mathrm{~b}$. It is worth noticing that although the SEA parameter is similar for Lattice and Foam, the differences between the EA parameters are more distinct. The Lattice structure has the highest EA parameter but the conducted tests do not allow for a precise comparison of the absorption properties, as Lattice has not reached the densification phase. The analysis shows also that the auxetic properties of the negative Poisson ratio possessed by the Auxcetic do not result in its improved performance in application to blast mitigation. The SEA parameter of the tested Auxetic is 2 to 5 times smaller when compared to Honeycomb or to the structures with $3 \mathrm{D}$ unit cells, respectively. 


\section{Conclusion}

The conducted blast compression tests of the additively manufactured samples resulted in a comparison of the dynamic behaviour of four geometries of Honeycomb, Auxetic, Lattice and Foam. The analysis of the mechanical deformations shows that the structures composed with the 3D unit cell absorb more energy during the blast loading carried out by means of the EDST set-up. Based on the calculated energy absorption characteristics, it may be concluded that despite that these samples are characterized by a higher mass, they mitigate more efficiently an explosive load than the structures with 2D unit cells.

The developed simulation, built on the example of Honeycomb analyses the deformation mechanism observed experimentally. The numerical results indicate non-strained areas which removal should not change the mechanism of the blast energy absorption but which may improve the SEA parameter. The conducted research provides a good basis for an analysis and a further geometry optimization of structures applied to attenuate explosive waves.

\section{References}

1. L. Blanc, M.-O. Sturtzer, T. Schunck, D. Eckenfels, J.-F. Legendre, Blast wave mitigation using multi-phase solid material in a sandwich cladding, in Proceedings of the $25^{\text {th }}$ Military Aspects of Blast and Shock, The Hague, NL, September 2018

2. A.S.M. AymanAshab, D. Ruan, G. Lu, A.A. Bhuiyan, Mater. 9, 162 (2016)

3. M. Yamashita, M. Gotoh, Int. J. Imp. Eng. 32, 618-630 (2005)

4. T. Fras Spr. Sci. B. Med., LLC 69, 1-30 (2020)

5. T. Fras, L. Colard, P. Pawlowski, Int. J. Multiphys. 9, 267-285 (2015)

6. S. McKown, Y. Shen, W.K. Brookes, C.J.Sutcliffe, W.J. Cantwell, G.S. Langdon, G.N. Nutrick, M.D. Theobald, Int. J. Imp. Eng 35, 795-810 (2008)

7. B. Branch, A. Ionita, B.E. Clements, D.S. Montgomery, B.J. Jensen, B. Patterson, A. Schmalzer, A. Mueller, D.M. Dattelbauma J. Appl. Phys. 121, 135102-1-125102-9 (2017)

8. EOS GmbH - Electro Optical Systems, AD, 1-4 (2010)

9. M. Moataz, L. N. C. Attallah, Q. Chunlei, R. Noriko, W. Wei, CRC Press, Taylor \& Francis 3, 59-110 (2018)

10. Z.H. Xionga, S.L. Liub, S.F. Lia, Y. Shic, Y.F. Yanga, R.D.K. Misrad, Mater. Sci. Eng. 156, 148-156 (2019)

11. J.R. Klepaczko, A. Rusinek, J.A. Rodríguez-Martínez, R.B. Pęcherski, A. Arias, Mech. Mater. 41, 599-621 (2009)

12. M. Stanczak, T. Fras, L. Blanc, P. Pawlowski, A. Rusinek Met. 9, 1350:1-24 (2019)

13. M.F. Ashby, L.J. Gibson, CUP, 93-174 (1997)

14. T. Wierzbicki, W. Abramowicz, J. Appl. Mech. 50, 727-734 (1983)

15. T. Fras, I. Szachogluchowicz, L. Sniezek, Eur. Phys. J. 227, 17-27 (2018)

16. M. Stanczak, T. Fras, L. Blanc, P. Pawlowski, A. Rusinek, Numerical modelling of honeycomb structure subjected to blast loading, in Proceedings of the 12th European Ls-Dyna Conference 2019, 13-16 May 2019, Koblenz, Germany (2019) 